\title{
Non-Monochromatic Electromagnetic Radiation of Inhomogeneous Media
}

\author{
Nicolay N. Grinchik ${ }^{1}$, Vladimir G. Andrejev², Galina M. Zayats ${ }^{3}$, Yury N. Grinchik ${ }^{4}$ \\ ${ }^{1}$ A. V. Luikov Heat and Mass Transfer Institute of the National Academy of Sciences of Belarus, Minsk, Belarus \\ ${ }^{2}$ Ryazan State Radio Engineering University, Ryazan, Russia \\ ${ }^{3}$ Institute of Mathematics of the National Academy of Sciences of Belarus, Minsk, Belarus \\ ${ }^{4}$ Minsk, Belarus \\ Email: nngrin@yandex.ru,Andrejev.V.G@rsreu.ru, zayats@im.bas-net.by, grinchik77@gmail.com
}

How to cite this paper: Grinchik, N.N., Andrejev, V.G., Zayats, G.M. and Grinchik, Yu.N. (2018) Non-Monochromatic Electromagnetic Radiation of Inhomogeneous Media. Journal of Electromagnetic Analysis and Applications, 10, 13-33.

https://doi.org/10.4236/jemaa.2018.102002

Received: December 1, 2017

Accepted: February 6, 2018

Published: February 9, 2018

Copyright $\odot 2018$ by authors and Scientific Research Publishing Inc. This work is licensed under the Creative Commons Attribution International License (CC BY 4.0).

http://creativecommons.org/licenses/by/4.0/

\begin{abstract}
A coordinated physicomathematical model for the propagation of a soliton-like electromagnetic pulse in a heterogeneous medium is developed in the presence of strong discontinuities in the electromagnetic field. The model is based on the reduction of Maxwell's equations to the well-studied wave equation. When the electromagnetic pulse was specified, its amplitude modulation was taken into account, as was the nonstationary broadening of the spectral line. Conditions for matching the momentum for the first initial boundary-value problem are obtained. The time dispersion of the electrical induction is taken into account in terms of the function of signal conditioning which takes account of the broadening of its spectral line and integration over the continuous spectrum. With this approach, it is not necessary to neglect spatial derivatives, and also to use spatial nonlocal relations to take account of the effect of surface charge, surface current, and spatial dispersion of electrical induction at the interfaces of adjacent media.
\end{abstract}

\section{Keywords}

Strong Discontinuities in the Electromagnetic Field, Nonstationary

Broadening of the Spectral Line, Surface Charge, Surface Current,

Electromagnetic Wave, Heterogeneous Medium, Soliton-Like

Electromagnetic Pulse, Induced Surface Charge

\section{Introduction}

In many fields of technology, for example, radar and biophysics, it is of interest to study signal propagation in a heterogeneous medium. Real pulse signals in most cases are not strictly monochromatic and are characterized by the width of 
spectral lines. In this case, in a heterogeneous medium, at the interfaces of adjacent media, as a rule, the function of the first kind is discontinuous, i.e., a strong discontinuity in the electromagnetic field.

In radio-physics, one often uses, for the problem of propagation of electromagnetic waves in the presence of "perfectly conducting" bodies, a surface impedance of the form as the boundary condition in determining outside the conductor (M.A. Leontovich, 1948); the fulfillment of the condition of continuity of the tangential components of the electric and magnetic field strength, i.e., the absence of a surface current, is assumed. Not only do the induced surface charge and current character the properties of the surface, but they are also functions of the process; therefore, surface impedances hold for conditions under which they have been determined. Under other experimental conditions, one cannot use surface impedances, since surface impedances depend on the structure of the determined field and are not boundary conditions in the ordinary sense. When the notion "surface impedance" is used there are a number of constraints and assumptions: the depth of penetration of an electromagnetic wave into a medium and the wavelength in it must be small compared to the wavelength in the ambient space, compared to the distances from the field's sources, and compared to the radii of curvature of the body's surface; the changes in the relative permittivity along the body's length and in the magnetic permeability at distance of the order of the wavelength (or on a length equal to the penetration depth) are small. Furthermore, if we have no ware absorption or it is minor, it is difficult to use the existing impedance conditions, since in the body, there can be waves traveling not only from the surface but from the body as well. By virtue of the aforesaid, the scattering of electromagnetic waves by a group of closely spaced bodies assumes the use of exact boundary conditions with introduction of a surface charge and a surface current.

\section{Statement of the Problem}

Let us consider the interface between two media with different electrophysical properties. On both sides, the vectors of magnetic field strength and magnetic induction, as well as electric field and electric displacement vectors are finite and continuous, but on the surface $S$ they can undergo a discontinuity of the first kind. Furthermore, induced surface charges $\sigma$ and surface currents i (vectors lying in the tangent plane to the surface $S$ ) arise at the interface under the action of an external electric field.

When studying the electric field interacting with the material medium, we use Maxwell's equations (1857)

$$
\begin{gathered}
\boldsymbol{j}_{\text {total }}=\nabla \times \boldsymbol{H}, \quad \nabla \boldsymbol{D}=\rho, \\
-\frac{\partial \boldsymbol{B}}{\partial t}=\nabla \times \boldsymbol{E}, \quad \nabla \boldsymbol{B}=0,
\end{gathered}
$$

where $\boldsymbol{j}_{\text {total }}=\lambda \boldsymbol{E}+\frac{\partial \boldsymbol{D}}{\partial t}, \quad \boldsymbol{B}=\mu \mu_{0} \boldsymbol{H}$, and $\boldsymbol{D}=\varepsilon \varepsilon_{0} \boldsymbol{E}$. 
The system of equations is supplemented, on the interface $S$, with the boundary conditions

$$
\begin{gathered}
D_{n_{1}}-D_{n_{2}}=\sigma, \\
E_{\tau_{1}}-E_{\tau_{2}}=0, \\
B_{n_{1}}-B_{n_{2}}=0, \\
\boldsymbol{H}_{\tau_{1}}-\boldsymbol{H}_{\tau_{2}}=\left[\boldsymbol{i}_{\tau} \boldsymbol{n}\right] .
\end{gathered}
$$

The subscripts $n$ and $\tau$ denote the normal and tangential components of the vectors to the surface $S$, and the subscripts 1 and 2, the adjacent media with different electrophysical properties. By $\tau$ we mean any direction tangential to the discontinuity surface. At the same time, there is no closing relation for the induced surface charge $\sigma$, which leads to the necessity of introducing an impedance matrix, which is determined experimentally or in some cases theoretically from quantum representations.

Not only does the induced surface charge $\sigma$ characterize the properties of the surface, but it is also is a function of the process, i.e., $\sigma(\boldsymbol{E}(\partial \boldsymbol{E} / \partial t, \boldsymbol{H}(\partial \boldsymbol{H} / \partial t)))$. Therefore the surface impedances are valid for conditions under which they were determined. Under other experimental conditions, it is impossible to use surface impedances. The problem of determining the surface charge and the surface current at the interfaces is further complicated by the study and modeling of nonstationary electrochemical processes and of pulsed electrolysis, since the surface charge is influenced by heat and mass fluxes. Let us show that $\sigma$ can be calculated from the phenomenological macroscopic equations of the Maxwell electromagnetic field and the law of conservation of electric charge taking account of the specific nature of the interface of adjacent media.

The Maxwell Equations (1), (2) represent a system of first-order differential equations containing unknown components of the field $\boldsymbol{E}(x, y, z ; t)$ and $\boldsymbol{H}(x, y, z ; t)$, where the spatial coordinates $x, y, z$ and the time parameter $t$ vary within the ranges $(x, y, z) \in D \subset R^{3}$ and $0 \leq t \leq \infty$. However, to determine the unknown vector functions in a rapidly varying field, it is expedient to exclude one field from Equations (1), (2) and to go to higher-order equations containing only the unknown field $\boldsymbol{E}$ or $\boldsymbol{H}$. This is due to the fact that the resulting equations in many cases are classical and well-studied.

We assume that the parameters of the medium $\varepsilon, \mu$ and $\gamma$ depend only on the coordinates $x, y, z$ and have sufficient smoothness, i.e., we consider piecewise homogeneous media.

A Generalized Wave Equation for $E$ and Conditions at the Interfaces in the Presence of Strong Discontinuities of the Electromagnetic Field. Formulation of the Physicomathematical Model of Propagation of the Electromagnetic Field in a Layered Medium

We multiply the left and right sides of the equation for the total current (1) by 
$\mu \mu_{0}$ and differentiate it with respect to time. We apply the curl operator to the left and right sides of Equation (2) and, setting $\mu \geq 1$-const, obtain

$$
\frac{\partial \boldsymbol{j}_{\text {total }}}{\partial t}=\frac{1}{\mu \mu_{0}} \nabla^{2} \boldsymbol{E}-\frac{1}{\mu \mu_{0}} \operatorname{grad}(\operatorname{div} \boldsymbol{E}) .
$$

In Cartesian coordinates (7), will have the form

$$
\begin{aligned}
& \frac{\partial j_{\text {totalx }}}{\partial t}=\frac{1}{\mu \mu_{0}}\left(\frac{\partial^{2} E_{x}}{\partial x^{2}}+\frac{\partial^{2} E_{x}}{\partial y^{2}}+\frac{\partial^{2} E_{x}}{\partial z^{2}}\right)-\frac{1}{\mu \mu_{0}} \frac{\partial}{\partial x}\left(\frac{\partial E_{x}}{\partial x}+\frac{\partial E_{y}}{\partial y}+\frac{\partial E_{z}}{\partial z}\right), \\
& \frac{\partial j_{\text {totaly }}}{\partial t}=\frac{1}{\mu \mu_{0}}\left(\frac{\partial^{2} E_{y}}{\partial x^{2}}+\frac{\partial^{2} E_{y}}{\partial y^{2}}+\frac{\partial^{2} E_{y}}{\partial z^{2}}\right)-\frac{1}{\mu \mu_{0}} \frac{\partial}{\partial y}\left(\frac{\partial E_{x}}{\partial x}+\frac{\partial E_{y}}{\partial y}+\frac{\partial E_{z}}{\partial z}\right), \\
& \frac{\partial j_{\text {total } z}}{\partial t}=\frac{1}{\mu \mu_{0}}\left(\frac{\partial^{2} E_{z}}{\partial x^{2}}+\frac{\partial^{2} E_{z}}{\partial y^{2}}+\frac{\partial^{2} E_{z}}{\partial z^{2}}\right)-\frac{1}{\mu \mu_{0}} \frac{\partial}{\partial z}\left(\frac{\partial E_{x}}{\partial x}+\frac{\partial E_{y}}{\partial y}+\frac{\partial E_{z}}{\partial z}\right) .
\end{aligned}
$$

On the interface, there is also the relation

$$
\operatorname{div} \boldsymbol{i}_{\tau}+I_{q x_{1}}-I_{q x_{2}}=-\frac{\partial \sigma}{\partial t}
$$

which reflects the law of conservation of charge at the interface of adjacent media. Equations (3)-(6) can be written in the Cartesian coordinate system:

$$
\begin{gathered}
D_{x_{1}}-D_{x_{2}}=\sigma, \\
E_{y_{1}}-E_{y_{2}}=0, \\
E_{z_{1}}-E_{z_{2}}=0, \\
B_{x_{1}}-B_{x_{2}}=0, \\
H_{y_{1}}-H_{y_{2}}=i_{z}, \\
H_{z_{1}}-H_{z_{2}}=i_{y} .
\end{gathered}
$$

where $\boldsymbol{i}_{\tau}=i_{y} \boldsymbol{j}+i_{z} \boldsymbol{k}$ is the surface current density, while the $x$ coordinate is directed along the normal to the interface. By the densities $i_{y}, i_{z}$ of surface currents, we mean the quantity of electricity flowing per unit time through a unit length of a segment located on the surface along which the current flows and perpendicular to the direction of the current.

The order of the system of differential Equations (8)-(10) is 18. Therefore, at the interface $S$, generally speaking, nine boundary conditions must be specified. In addition, three more conditions (12), (16), and (17) containing unknown (before the solution) quantities must be satisfied at this boundary. Therefore, the total number of conjugation conditions at the interface $S$ must be equal to 12 for correct solution of the problem.

Differentiating the expression of Equation (12) with respect to time and taking into account relation (11), at the interface, we obtain the condition for the normal components of the total current:

$$
\operatorname{div} \boldsymbol{i}_{\tau}+j_{\text {total }_{1}}=j_{\text {total }_{2}},
$$

which makes it possible to exclude from consideration the surface charge densi- 
ty.

Due to the equality of tangential projections of the electric field in $z$ and $y$, according to Equations (13), (14), the expressions for the surface current densities $\boldsymbol{i}_{z}$ and $\boldsymbol{i}_{y}$ have the form

$$
i_{z}=\left.\bar{\lambda} E_{z}\right|_{x=\xi}, i_{y}=\left.\bar{\lambda} E_{y}\right|_{x=\xi}
$$

where

$$
\bar{\lambda}=\left.\frac{1}{2}\left(\lambda_{1}+\lambda_{2}\right)\right|_{x=\xi}
$$

is the mean value of electrical conductivity at the interfaces $\zeta$ of adjacent media in accordance with the Dirichlet theorem for a piecewise smooth and piecewisedifferentiable function. Consequently, it follows from Formulas (19) and (20) that

$$
\left.\left[\operatorname{divi} \mathbf{i}_{\tau}\right]\right|_{x=\xi}=0
$$

Relation (21) and, as a consequence, the equality of the normal components of the total current were obtained by another method in 1948 in the work of G.A. Grinberg and V.A. Fock. It was shown in [1]-[6] that Equation (21) leads to the equality of the derivatives of the electric field strength along the normal to the surface:

$$
\left.\left[\frac{\partial E_{x}}{\partial x}\right]\right|_{x=\xi}=0
$$

Earlier, in [1]-[6], we obtained and formulated all fifteen conditions at the interface of adjacent media necessary for solving the complete system of Equations (8)-(10):

1) The equality of normal components of the total current;

2) The equality of tangential projections of the vortex of the electric field;

3) The law of conservation of electric charge;

4) The equality of tangential components of the electric field and their derivatives in the tangential direction;

5) The equality of the derivatives of normal components of the total current in a direction tangential to the interface of adjacent media, with account of the effect of surface currents without introducing explicitly the surface charge $\sigma$.

These conditions are valid in each cross section of the sample under study. For numerical modeling of the interaction of an electromagnetic field and a heterogeneous medium, it is most expedient to use, in our opinion, the through-counting schemes [1]-[6], since the continuity condition of the total current must be satisfied at the interfaces of adjacent media. Indeed, in solving electro-dynamics problems, according to Equations (1)-(6), it is necessary to set six conditions on each face. Therefore, in 2D we must already have 24 conditions. Furthermore, on "sharp" faces, i.e., at angular points in Cartesian coordinates, it is necessary to additionally specify the Sommerfeld conditions in the asymptotic approximation of Ma- 
lyuzhinets. Thus, to solve the problem even for one object, we must have 28 conditions. It is clear that for a group of bodies, it is technically quite difficult to realize the task.

Furthermore, Maxwell's boundary conditions do not contain any closing relations for the induced surface charge and surface current. The use of known Leontovich-Shchukin conditions at interfaces, for example, for nanoobjects, is incorrect, since these conditions assume that the electrophysical properties and characteristics of the object vary little over distances of the order of a wavelength. The surface charge due to the passage of a direct current through the interface of adjacent media can be determined according to [1] [4] [5] [6] [7]:

$$
\sigma=(U / R S) \varepsilon_{0}\left[\left(\varepsilon_{1} / \lambda_{1}\right)-\left(\varepsilon_{2} / \lambda_{2}\right)\right] .
$$

As follows from (22), the induced electric charge $\sigma$ is determined by the magnitude of the current, and also by a factor that takes into account the properties of the medium. In textbooks [8]-[14], it is assumed that the surface charge is zero and is associated with electrostatics. As can be seen from Formula (22a), this assertion is valid only if

$$
\left[\left(\varepsilon_{1} / \lambda_{1}\right)-\left(\varepsilon_{2} / \lambda_{2}\right)\right]=0
$$

The reformulation of Maxwell's equations and their reduction to a wave equation make it possible to exclude from consideration surface charge and current [1]-[6], but in this case the number of boundary conditions becomes significantly larger, since at each boundary it is required to specify nine first derivatives now with respect to coordinates, as well as $E_{x}, E_{y}$, and $E_{z}$, and their derivatives with respect to time, i.e. 15 conditions, and the problem also becomes technically unrealizable. The way out is to use the continuity condition of the total current and through-counting schemes [1]-[6]. The calculation method consists in that the investigated region containing various inhomogeneous (angular, layered) structures is conventionally placed in the "casing" and we specify the boundary conditions only on it; usually, they are functions of the form $E_{x}=E_{x}(t) \sin \omega t$ and $E_{y}=E_{y}(t) \cos \omega$. The medium inside the "casing" is considered together with inhomogeneous inclusions as a single medium, when its properties depend on the coordinates, i.e., $\varepsilon(\boldsymbol{r}), \lambda(\boldsymbol{r})$. This approach was used by us earlier to simulate the interaction of an electromagnetic wave and macro objects using the Comsol Multiphysics package [4] [6]. The characteristic dimension of the "casing" must be greater than the characteristic dimension of the object so that the reflected wave does not affect the boundary conditions. The proposed method also proved to be workable as applied to nanoobjects measuring $10-50 \mathrm{~nm}$, wavelength $\sim 400-500 \mathrm{~nm}$ (light). Note that the Comsol Multiphysics software package specifically focused on solution of electrodynamics problems. Unfortunately, using this package, as well as other software packages for solving electrodynamics problems, it is possible to obtain unreliable results, since the solution does not require matching conditions at angular points for the initial boun- 
dary-value problem. For clarity, we consider the one-dimensional wave equation of oscillations of a string:

$$
\begin{gathered}
\frac{\partial^{2} u}{\partial t^{2}}-a^{2} \frac{\partial^{2} u}{\partial x^{2}}=f(x, t), \quad(x, t) \in \bar{D}, \\
\left.u\right|_{t=0}=\varphi(x),\left.\frac{\partial u}{\partial t}\right|_{t=0}=\psi(x), 0 \leq x \leq l, \\
\left.u\right|_{x=0}=\mu_{1}(t),\left.u\right|_{x=l}=\mu_{2}(t), t \geq 0 .
\end{gathered}
$$

With given functions $f_{x x}^{\prime \prime} \in C(\bar{D}), \varphi \in C^{2}(0 \leq x \leq l), \psi \in C^{1}(0 \leq x \leq l)$, and $\mu_{1} \in C^{2}(0 \leq x \leq \infty)$, you need to find a function $u \in C^{2}(\bar{D})$, that satisfies Equation(23) in the region $\bar{D}$, initial conditions (24), and boundary conditions of the first kind (25). Problem (23)-(25) describes the process of oscillations of a homogeneous string of length $l$ stretched along a segment $0 \leq x \leq l$. The first initial condition of (24) defines the graph $u=\varphi(x)$ of the string at the initial time $t=0$, and the quantity $\psi(x)$ from the second initial condition of (24) is the initial velocity of the string at the point with a coordinate $x$.

Note that when formulating the problem (23)-(25), certain restrictions must be imposed on the given functions $\varphi, \psi$, and $\mu_{1}$. In particular, matching conditions must be satisfied at the angular points of the region [15] [16]:

$$
\varphi(0)=\mu_{1}(0), \varphi(l)=\mu_{2}(0), \mu_{1}^{\prime}(0)=\psi(0), \mu_{2}^{\prime}(0)=\psi(l) .
$$

These conditions are necessary conditions for the continuous differentiability of the solution $u(x, t)$ in a closed region $\bar{D}$. Since the solution is $u \in C^{2}(\bar{D})$, then in addition to conditions (26), second-order conditions must be satisfied:

$$
\mu_{1}^{\prime \prime}(0)-a^{2} \varphi^{\prime \prime}(0)=f(0,0), \mu_{2}^{\prime \prime}(0)-a^{2} \varphi^{\prime \prime}(l)=f(l, 0) .
$$

Indeed, we differentiate conditions (25) twice with respect to $t$, and the first condition of (24), twice with respect to $x$, then

$$
\begin{aligned}
& \left.\frac{\partial^{2} u}{\partial t^{2}}\right|_{x=0, t=0}=\mu_{1}^{\prime \prime}(0),\left.\frac{\partial^{2} u}{\partial t^{2}}\right|_{x=l, t=0}=\mu_{2}^{\prime \prime}(0), \\
& \left.\frac{\partial^{2} u}{\partial x^{2}}\right|_{x=0, t=0}=\varphi^{\prime \prime}(0),\left.\frac{\partial^{2} u}{\partial x^{2}}\right|_{x=l, t=0}=\varphi^{\prime \prime}(l) .
\end{aligned}
$$

Substituting the values of the derivatives at the corresponding points to Equation (23), we obtain the required conditions (27) [15] [16].

In the future, we will be interested in waves generated by sources that perform modulated oscillations:

$$
A \cos w t \pm \frac{m A}{2} \cos w_{1} t \pm \frac{m A}{2} \cos w_{2} t,
$$

where $w_{1}=w+\Omega$ and $w_{2}=w+\Omega$. Expression (29) is easily transformed into

$$
S=A[1 \pm m \cos (\Omega t-K x)] \cos (w t-K x)
$$

or 


$$
S=A[1 \pm m \cos (\Omega t-K x)] \sin (w t-K x)
$$

where $K=0.5\left(k_{1}-k_{2}\right), K^{\prime}=0.5\left(k_{1}+k_{2}\right)$, and $\Omega \ll w, \Omega$ is the frequency and $k_{1}$ and $k_{2}$ are the wave numbers.

In fact, the wave generated by our modulated source does not differ from the superposition of the three waves that would be created by three independent sources. Let us imagine that waves of the form (30) and (31), which were formed with the help of special "tuning forks", i.e. technical means, begin to propagate in a continuous medium. In this case, because of the interaction of a "direct" wave of the form (30) and (31) with the backward wave, the spectral line broadens. The broadening of the spectral line is explained by many effects: spin-spin interaction, signal absorption by the medium, exchange interaction, collision broadening, Doppler effect, etc. [17] [18] [19]. As is known, the general solution of the wave Equation (23) is the d'Alembert solution: the sum of the forward and backward waves, with the backward wave having a broadening of $\pm 2 \Delta$. As a result, we obtain for an electromagnetic wave

$$
\begin{aligned}
E_{x}(t) & =A[1+m \cos \Omega t][2 \sin w t-\sin (w-2 \Delta) t-\sin (w+2 \Delta) t] \\
& =4 A[1 \pm m \cos \Omega t] \sin ^{2} \Delta t \sin w t,
\end{aligned}
$$

where the frequency $w$ corresponds to a wave moving to the right, and the frequency $w \pm 2 \Delta$ corresponds to a wave moving to the left. A similar expression can also be obtained for $E_{y}$ :

$$
\begin{aligned}
E_{y}(t) & =A[1 \pm m \cos \Omega t][2 \cos w t-\cos (w-2 \Delta) t-\cos (w+2 \Delta) t] \\
& =4 A[1 \pm m \cos \Omega t] \sin ^{2} \Delta t \cos w t .
\end{aligned}
$$

In deriving (32) and (33), we used the well-known trigonometry formulas [20]:

$$
\begin{aligned}
\sin \alpha \sin \beta \sin \gamma= & \frac{1}{4}[\sin (\alpha+\beta-\gamma)+\sin (\beta+\gamma-\alpha) \\
& +\sin (\gamma+\alpha-\beta)-\sin (\alpha+\beta+\gamma)], \\
\sin \alpha \sin \beta \cos \gamma= & \frac{1}{4}[-\cos (\alpha+\beta-\gamma)+\cos (\beta+\gamma-\alpha) \\
& +\cos (\gamma+\alpha-\beta)-\cos (\alpha+\beta+\gamma)]
\end{aligned}
$$

with the condition $\alpha=\beta$.

The broadening of the spectral lines is due to the interaction of the emitting atom with the surrounding particles: other atoms and molecules, ions and electrons. Therefore, functions of the form (32) and (33) continuously fill the frequency band $w-2 \Delta \leq w \leq w+2 \Delta$. In addition, it takes some time to establish a signal (transient process), finally, for the components $E_{x}$ and $E_{y}$ we have

$$
\begin{aligned}
E_{x}(0, t)= & A\left[\int_{w-2 \Delta}^{w+2 \Delta}\left(1-\frac{2}{\mathrm{e}^{\lambda \sin ^{2} \varphi t}+\mathrm{e}^{-\lambda \sin ^{2} \varphi t}}\right) \sin ^{2} \varphi t \mathrm{~d} \varphi\right] \\
& \times \frac{(1-m \cos \sigma t) \sin w t}{4 \Delta},
\end{aligned}
$$




$$
\begin{aligned}
E_{y}(0, t)= & A\left[\int_{w-2 \Delta}^{w+2 \Delta}\left(1-\frac{2}{\mathrm{e}^{\lambda \sin ^{2} \varphi t}+\mathrm{e}^{-\lambda \sin ^{2} \varphi t}}\right) \sin ^{2} \varphi t \mathrm{~d} \varphi\right] \\
& \times \frac{(1-m \cos \sigma t) \cos w t}{4 \Delta} .
\end{aligned}
$$

It is easy to verify that $E_{x}(0, t)$ and $E_{z}(0, t)$ satisfy the necessary matching conditions, since $E_{x}(0, t)=0, E_{x}^{\prime}(0, t)=0, E_{z}(0, t)=0$, and $E_{z}^{\prime}(0, t)=0$, when the pulse propagates into a medium with zero initial conditions. A group of waves is a kind of oscillatory circuit with distributed parameters, in which "forced" oscillations are not established immediately, but only after a certain time after "including an external emf" [8].

At the initial time moment, when $t \rightarrow 0$, the expression in parentheses in the integrand expressions (34), (35) exactly corresponds to the "establishment function" in the oscillatory circuit under the action of an external sinusoidal electromotive force [8].

Below we confine ourselves to linear electrodynamics. In this case the constitutive equations are linear relations. For constant fields, the constitutive equations corresponding to Equations (1), (2) can be written in the form

$$
D_{i}=\varepsilon_{i j} E_{j}, B_{i}=\mu_{i j} H_{j} .
$$

Here $\varepsilon_{i j}$ and $\mu_{i j}$ are the tensors of permeability and magnetic permeability. They are determined by the specific properties of the medium and thereby characterize its electromagnetic properties. Such constitutive equations hold only for rather slowly varying fields. For fast-changing fields that change rapidly compared to the characteristic relaxation time in the medium or to the periods of characteristic natural oscillations of the medium, the situation becomes somewhat more complicated. In this case, the state of the medium turns out to depend not only on the field at a given instant $t$, but also on its values at the preceding instants of time. This can be understood if we take into account, for example, the fact that the relaxation process that has begun in the medium under the action of a field that appeared at the time $t$ and vanished after a certain time, which is much shorter than the relaxation time, will still flow after the field disappears. Therefore, for high-frequency fields, it is necessary to use constitutive equations of the form [21]

$$
\begin{aligned}
& D_{i}(t)=\int_{-\infty}^{t} \mathrm{~d} t^{\prime} \hat{\varepsilon}_{i j}\left(t-t^{\prime}\right) E_{j}\left(t^{\prime}\right), \\
& B_{i}(t)=\int_{-\infty}^{t} \mathrm{~d} t^{\prime} \hat{\mu}_{i j}\left(t-t^{\prime}\right) H_{j}\left(t^{\prime}\right) .
\end{aligned}
$$

Relations (36) take into account the influence of prehistory on the electromagnetic properties of the medium. In this case, we usually speak of the time or frequency dispersion.

It is quite natural to raise the question of why, for example, the electric induction in relations (36) is considered to depend only on the electric field. In principle, one could speak of the dependence of $\boldsymbol{D}$ on the magnetic induction $\boldsymbol{B}$. However, in the latter case, using the field equation 


$$
\operatorname{rot} \boldsymbol{E}=-\frac{1}{c} \frac{\partial \boldsymbol{B}}{\partial t},
$$

it would be possible to eliminate the quantity $\boldsymbol{B}$. In this case, although the electric induction would again be expressed only by the electric field strength, the spatial derivatives of $\boldsymbol{E}$ would arise in this constitutive equation. Therefore, we can say that the constitutive Equations (36) are valid only when the spatial derivatives can be neglected. In other words, such constitutive equations are valid for fields that vary slowly in space [21].

When functions of the form (34) and (35) are used the relaxation nature of the process in a rapidly varying field is taken into account by us as a transition function, which is an integral not only in frequency but in time and space after numerical integration of the wave equation. This remark applies not only to temporal, but also to spatial dispersion. According to [21], for fields that change sharply in space, it is obviously necessary to take into account the influence of the field at remote points on the electromagnetic properties of the medium at a given point in space. Indeed, for example, because of the transport processes, the state at a certain point in the medium will be assigned not only by the value of the field at this point, but also by the field in entire regions of the medium, from which the field effect is transferred as a result of the transfer of matter. Therefore, instead of the constitutive Equations (36), it is necessary to use spatially nonlocal relations that take into account not only temporal, but also spatial dispersion. For homogeneous isotropic and nonisotropic media, such relations can be written in the following form [21]:

$$
\begin{aligned}
& \boldsymbol{D}(\boldsymbol{r}, t)=\int_{-\infty}^{t} \mathrm{~d} t^{\prime} \int \mathrm{d} \boldsymbol{r}^{\prime} \hat{\varepsilon}\left(t-t^{\prime},\left|\boldsymbol{r}-\boldsymbol{r}^{\prime}\right|\right) \boldsymbol{E}\left(\boldsymbol{r}^{\prime}, t^{\prime}\right), \\
& \boldsymbol{B}(\boldsymbol{r}, t)=\int_{-\infty}^{t} \mathrm{~d} t^{\prime} \int \mathrm{d} \boldsymbol{r}^{\prime} \hat{\mu}\left(t-t^{\prime},\left|\boldsymbol{r}-\boldsymbol{r}^{\prime}\right|\right) \boldsymbol{H}\left(\boldsymbol{r}^{\prime}, t^{\prime}\right) .
\end{aligned}
$$

Thus, the electromagnetic properties of such a medium are determined by two functions dependent on $\boldsymbol{r}$ and $t$. When spatial dispersion is taken into account, in accordance with (37), the vector functions $\boldsymbol{D}$ and $\boldsymbol{B}$ can be determined by solution of the integral-differential Equations (1), (2), and (37). In this case, only harmonic functions are considered in the paper without taking into account the modulation and broadening of the spectral line. According to the popular opinion, any uniformly continuous function can be reduced to a trigonometric series by means of an expansion in a Fourier series, i.e., to harmonic functions. However, the functions (34) and (35) containing the inverse hyperbolic cosine are not actually expanded in a Fourier series (message of the Wolfram Mathematics package). Note that for the Fourier expansion we are to calculate integrals of the form:

$$
-\int_{\pi}^{\pi} f(x) \cos (n x) \mathrm{d} x, \quad \int_{-\pi}^{\pi} f(x) \sin (n x) \mathrm{d} x,
$$

where $f(x)$ is defined by Formulas (34) and (35); numerically they are calculated problematically, in view of the properties of the integrand, there is no analytical solution. 
The most significant applications of the theory of electromagnetic field have been obtained in radio engineering, when the field functions are harmonicly dependent on time, i.e.,

$$
\begin{aligned}
\boldsymbol{E}(\boldsymbol{r}, t) & =\boldsymbol{E}_{1}(\boldsymbol{r}) \cos w t+\boldsymbol{E}_{2}(\boldsymbol{r}) \sin w t, \\
\boldsymbol{H}(\boldsymbol{r}, t) & =\boldsymbol{H}_{1}(\boldsymbol{r}) \cos w t+\boldsymbol{H}_{2}(\boldsymbol{r}) \sin w t .
\end{aligned}
$$

Passing to the complex domain and introducing complex quantities $\boldsymbol{E}(\boldsymbol{r})=\boldsymbol{E}_{1}(\boldsymbol{r})+i \boldsymbol{E}_{2}(\boldsymbol{r})$ and $\boldsymbol{H}(\boldsymbol{r})=\boldsymbol{H}_{1}(\boldsymbol{r})+i \boldsymbol{H}_{2}(\boldsymbol{r})$ and the complex dielectric constant of the medium, we exclude from Maxwell's equations the time parameter $t$ and determine the real electromagnetic field by means of the formulas

$$
\boldsymbol{E}=\operatorname{Re}\left(\boldsymbol{E} \mathrm{e}^{-i w t}\right), \quad \boldsymbol{H}=\operatorname{Re}\left(\boldsymbol{H e}^{-i w t}\right),
$$

where $\operatorname{Re}(\cdot)$ is the real part of the complex quantity in brackets.

Note that for an arbitrary function $\boldsymbol{E}(t)$, for example, (34) and (35), it is difficult to represent it in the form $E(t)=a(t) \cos \varphi(t)$, since the amplitude $a(t)$ and phase $\varphi(t)$ cannot be uniquely determined and it is not clear how to decompose $E(t)$ into the factors $a$ and $\cos \varphi$. Even greater difficulties arise in the case of transition to the complex representation $W(t)=U(t)+i V(t)$ when the real oscillation $E(t)$ is supplemented with the imaginary part $V(t)$. Problems arising in this case have been considered in detail in [22]. In this paper, it is emphasized that without defining uniquely amplitude, phase, and frequency, some methods using complex representation and claiming increased accuracy become meaningless.

We do not know of papers using (37) and in which solutions of the problems even for a homogeneous medium are given. However, it is not clear how to take into account temporal and spatial dispersion at the interfaces of adjacent media in the presence of strong discontinuities in the electromagnetic field. With this phenomenological approach in constitutive equations, it is necessary to take into account, for example, the dependence not only on frequency, but also the broadening of the spectral line $\Delta$, as well as the parameter that determines the establishment of the vibration $\lambda$.

\section{Numerical Simulation}

The method of the "non-sharp" boundary is substantiated using the Dirichlet theorem for piecewise smooth functions and the condition of continuity of the total current. Numerical simulation of the propagation of a soliton-like pulse signal in a medium with strong electromagnetic field discontinuities is performed with account taken of the broadening of the spectral line.

\subsection{Features of Calculating the Propagation of Electromagnetic Waves in Angular Structures. Analysis of a Soliton-Like Pulse Signal}

Electromagnetic phenomena arising from the incidence of plane electromagnetic waves on the interface of different media play an important role in engineering, 
since all real devices are bounded by surfaces and are inhomogeneous in space. With the help of the proposed physicomathematical model, it is possible to investigate the transmission of an electromagnetic wave by a layered angular structure.

In connection with the fact that in every cross section of the layered medium, the condition of continuity of the total current is valid, we use the through-counting schemes without identifying explicitly the interface of adjacent media. The procedure of calculation of $E_{x}$ at the interface is as follows. Since $E_{x_{1}} \neq E_{x_{2}}, E_{x}(x)$ experiences a discontinuity of the first-kind function (strong electromagnetic field discontinuity). Let us determine the value of the electric field strength at the point of discontinuity for $x=\xi$ on condition that $E_{x}(x)$ is piecewise smooth and piecewise differentiable, i.e., it has finite one-sided derivatives $E_{x^{\prime}}^{\prime \prime}(x)$ and $E_{x^{\prime \prime}}^{\prime \prime}(x)$. At the points of discontinuity $x_{i}$, we have

$$
\begin{aligned}
& E_{x^{+}}^{\prime}\left(x_{i}\right)=\lim _{\Delta x_{i} \rightarrow+0} \frac{E\left(x_{i}+\Delta x_{i}\right)-E\left(x_{i}+0\right)}{\Delta x_{i}}, \\
& E_{x^{-}}^{\prime}\left(x_{i}\right)=\lim _{\Delta x_{i} \rightarrow-0} \frac{E\left(x_{i}+\Delta x_{i}\right)-E\left(x_{i}-0\right)}{\Delta x_{i}} .
\end{aligned}
$$

In this case, according to the Dirichlet theorem for a uniformly continuous function $E(x)$, at the discontinuity point $\xi$, we have:

$$
E_{x=\xi}=\frac{1}{2}[E(\xi-0)+E(\xi+0)] \text {. }
$$

The Dirichlet condition also has a physical interpretation. When two solid conductors, dielectrics, and electrolytes come in contact in different combinations: metal-electrolyte, dielectric-electrolyte, metal-vacuum, etc., a double electric layer (DEL) is always formed at the interface of adjacent media, whose structure is usually unknown, but it significantly affects the electrokinetic phenomena, the rate of electrochemical processes, etc. It is important to note that in actual practice the electrophysical characteristics of $E(x)$ in the DEL are continuously changing, so Equation (38) is valid for the case where the thickness of the DEL, i.e., the thickness of the interphase boundary, is much smaller than the characteristic dimension of the homogeneous medium. In the case of a composite, for example, a metal with impregnations of dielectric balls, at a sufficiently high concentration of both components and smallness in their characteristic dimensions, the interphase boundaries overlap and condition (38) may be violated.

If the thickness of the DEL is much smaller than the characteristic dimensions $L$ of the objects under consideration, then Equation (38) also follows from the condition of linear variation in $E(x)$ in the region of the DEL. In actual practice, the thickness of the DEL depends on the kind of contacting substances and can be tens of angstroms. According to modern concepts, the outer shell of the DEL consists of two parts: the first is formed by ions closely drawn to the surface of the metal ("dense" or "Helmholtz" layer of thickness $h$ ), and the second, by 
ions located at distances from the surface exceeding the radius of the ion, with the number of these ions decreasing with distance from the interface ("diffuse layer"). The distribution of the potential in the dense and diffuse parts of the DEL is in fact exponential [23], i.e., the linearity condition $E(x)$ is violated. At the same time, the sum of the charges of the dense and diffuse parts of the outer shell of the DEL is equal to the charge of the inner lining of the DEL of the metal surface. However, if the thickness of the DEL is much smaller than the characteristic dimension of the object, then the expansion of $E(x)$ in a power series is valid, and we can confine ourselves to a linear approximation. According to the more general Dirichlet theorem (1829), the physical interpretation and knowledge of the $E(x)$ dependences in the region of the DEL are not required in the justification of Equation (38). Nevertheless, the above-mentioned known physical features of the DEL confirm the validity of fulfillment of condition (38).

The concept of surface charge has only an auxiliary meaning and was introduced for convenience in considering the field of charges at distances sufficiently large in comparison with the dimensions of the charges themselves. By studying the field at the adjacent media interfaces, i.e., near or inside the charges, we must return to the concept of the volume distribution of charges when $\operatorname{div} E \neq 0$, and the vector $\boldsymbol{E}$ is finite and continuous. In real physical processes, there is a double electrical layer at the interfaces of adjacent media and there is no disruption of the normal component of the electric field strength.

Thus, with account taken of the foregoing and of the validity of formula (38), in each cross section of the layered medium, for numerical solution it is expedient to use cross-counting schemes and at the same time to discretize the medium in such a way that the boundaries of the layers have common nodes.

For numerical simulation, we used the Wolfram Mathematics software package. We split the medium into finite elements in such a way that the nodes of the finite element mesh, lying on the interface, simultaneously belonged to media with different electrophysical properties. In this case, the condition of equality of the total currents and equality of the charge flows must be satisfied at the interface.

\subsection{Analysis of Pulsed Soliton-Like Signals}

We have considered waves of the following form, which will henceforth be called integrated

$$
\begin{aligned}
& E_{x}(t)=\frac{(1-m \cos \theta t) \sin \omega t}{4 \Delta} \int_{\omega-2 \Delta}^{\omega+2 \Delta}\left(1-\frac{2}{\mathrm{e}^{\lambda \sin ^{2} \varphi t}+\mathrm{e}^{-\lambda \sin ^{2} \varphi t}}\right) \sin ^{2} \varphi t \mathrm{~d} \varphi, \\
& E_{y}(t)=\frac{(1-m \cos \theta t) \cos \omega t}{4 \Delta} \int_{\omega-2 \Delta}^{\omega+2 \Delta}\left(1-\frac{2}{\mathrm{e}^{\lambda \sin ^{2} \varphi t}+\mathrm{e}^{-\lambda \sin ^{2} \varphi t}}\right) \sin ^{2} \varphi t \mathrm{~d} \varphi,
\end{aligned}
$$

as well as waves that will be called ordinary: 


$$
\begin{aligned}
& E_{x}(t)=\left(1-\frac{2}{\mathrm{e}^{\lambda \sin ^{2} \omega t}+\mathrm{e}^{-\lambda \sin ^{2} \omega t}}\right) \sin ^{2} \omega t(1-m \cos \theta t) \sin \omega t, \\
& E_{y}(t)=\left(1-\frac{2}{\mathrm{e}^{\lambda \sin ^{2} \omega t}+\mathrm{e}^{-\lambda \sin ^{2} \omega t}}\right) \sin ^{2} \omega t(1-m \cos \theta t) \cos \omega t,
\end{aligned}
$$

where $\omega=10^{9}, \theta=10^{6}, \Delta=10^{4}$, and $\lambda=0.1$. Let us consider the difference between the integrated and non-integrated waves, and also consider the integrated waves for different parameters $m$.

The graph of ordinary waves (component of the sine wave) with $t \in\left[2 \times 10^{-6}, 5 \times 10^{-5}\right]$ is shown in Figure 1 (a). As can be seen from the graph, these waves satisfy the principle of the transient process and are of an impulse nature. It is also worth noting that near zero, they are very close to 0 . Next, we consider the integrated waves on the same segment (component of the sine) (Figure 1(b)) and also consider in detail the shape of the wave crests (component of the sine), reducing the segment to $t \in\left[2 \times 10^{-6}, 5 \times 10^{-5}\right]$.

Let us represent the graphs of the same waves for the same parameters, but for the component of the cosine (Figure 2). Obviously, ordinary waves have a sinusoidal shape upon close examination. As could be seen from the graphs above, the integrated waves change their shape. Waves become "impulsive", which is a significant characteristic of waves, for example, for radar problems. In this case necessary conditions for coordination are fulfilled. The penetrating power of such a pulse in complex media, for example, the ionosphere and an

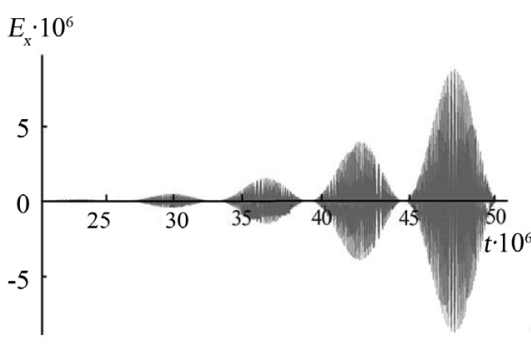

(a)

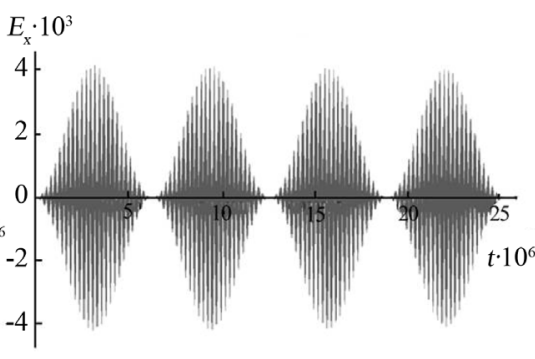

(b)

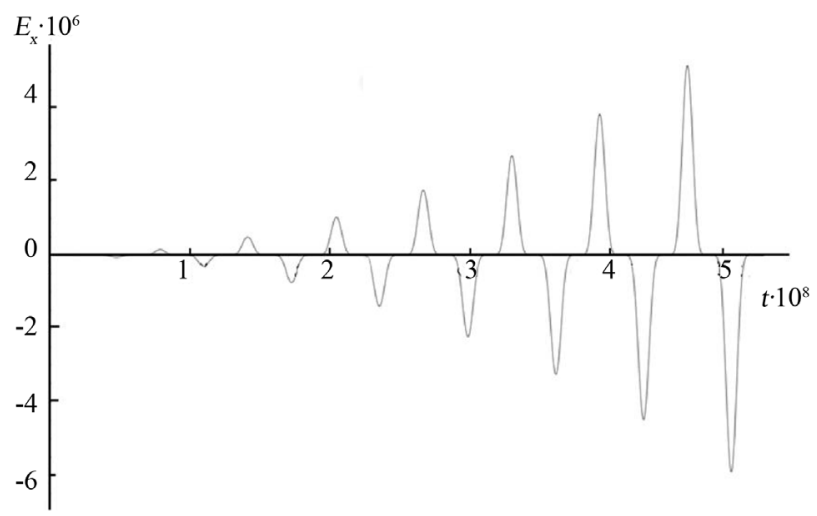

(c)

Figure 1. Graph of ordinary waves (component of the sine) (a) and graphs of integrated waves (components of the sine), $m=1$, different time scales (b and c). 
ust be much higher than for a rectangular and saw-tooth signal or other form of signal when necessary conditions for coordination are not fulfilled and as a consequence, we have the expenditure of energy on "noise" due to discontinuities in the solution of differential equations at the interface of adjacent media.

Let us consider the energy of ordinary waves and, by reducing the scale, the energy of the integrated waves is more detail (Figure 3). It can be seen from the figure that the qualitative characteristics of waves after integration are modified. The rate of increase in the electromagnetic pulse of the integrated waves also becomes several orders of magnitude higher. This means that the penetrating power of the integrated waves will be much higher than that of ordinary waves.

The exchange of information between cells in living organisms (electrolytes) occurs with the help of soliton-like pulses [24], therefore, it can be expected that these pulses may prove to be promising for signal transmission in the ionosphere and plasma.

We consider the integrated waves for different values of the parameter $m$ (Figure 4). The figure shows only the components of the sine for $m=0.7,1.0$, and 1.3. As noted earlier, at $m=1.0$, waves after a certain period are damped (go to zero). As the parameter $m$ decreases, the wave in the damping regions is smoothed out and no longer has a total attenuation, but only decreases to a certain limit. When the parameter $m$ increases at the damping points, a new wave crest appears which breaks the damping region into two new ones, smaller

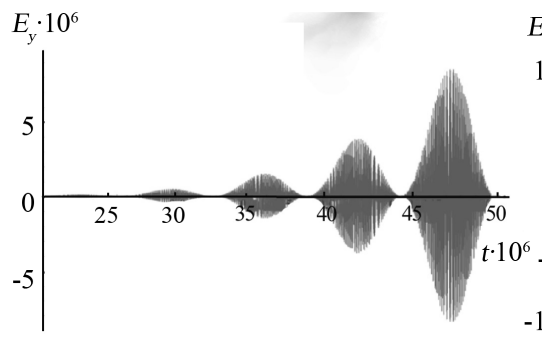

(a)

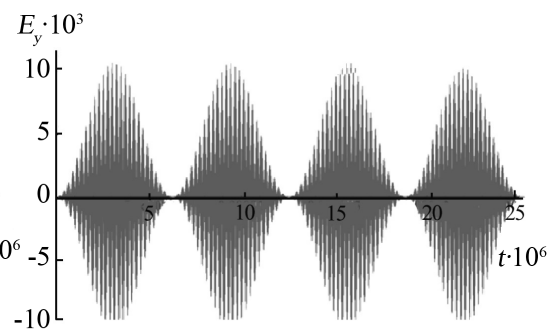

(b)

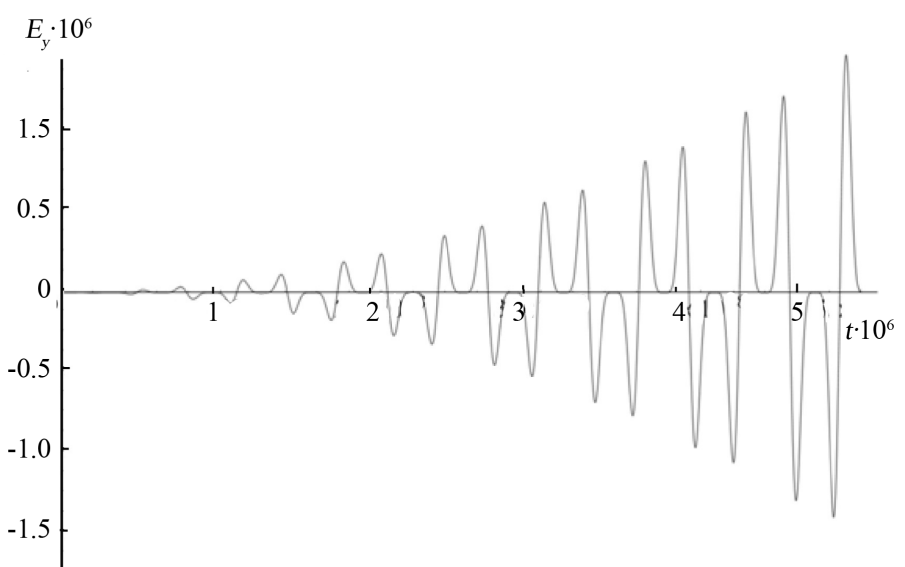

(c)

Figure 2. Graph of ordinary waves (component of the cosine) (a) and graphs of integrated waves (components of the cosine), $m=1$, different time scales (b and $c$ ). 


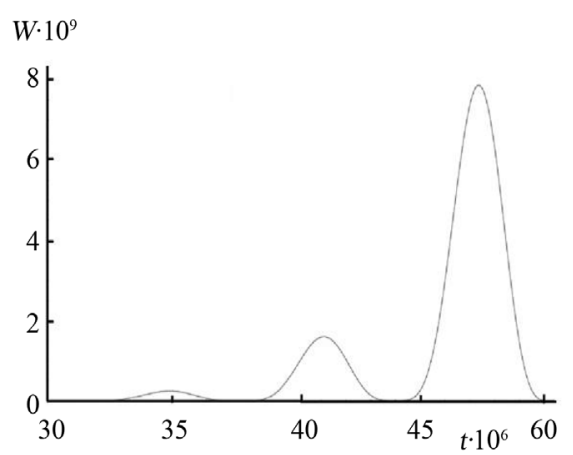

(a)

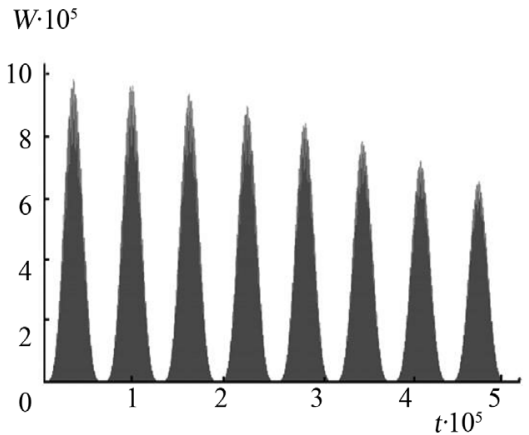

(b)

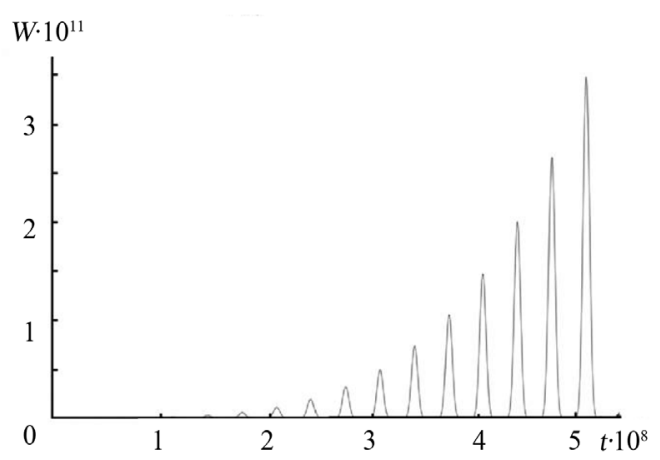

(c)

Figure 3. Graph of the energy of ordinary waves (a) and graphs of integrated waves for different time scales (b and c).

in size. It is also worth noting that the drawn conclusions are valid for the components of the cosine and for the energy and all components for ordinary waves.

\subsection{Numerical Simulation of an Electromagnetic Field in a Heterogeneous Medium}

The obtained waves can be used to solve the problem of their propagation in a medium with strong field discontinuities. Imagine that we have a square domain with dimensions $[0 ; 1] \times[0 ; 1]$. At the center of the domain, there is a copper bar (parallelepiped) (Figure 5).

It is necessary to determine how the intensity of the electromagnetic field varies in the given region. Earlier, we obtained a mathematical model that describes this process using differential equations, namely:

$$
\begin{gathered}
\lambda \frac{\partial E}{\partial t}+\varepsilon \varepsilon_{0} \frac{\partial^{2} E}{\partial t^{2}}=\frac{1}{\mu \mu_{0}}\left(\nabla^{2} E-\operatorname{grad}(\operatorname{div} E)\right), \\
\left.E\right|_{t=0}=0, \\
\left.\frac{\partial E}{\partial t}\right|_{t=0}=0,
\end{gathered}
$$



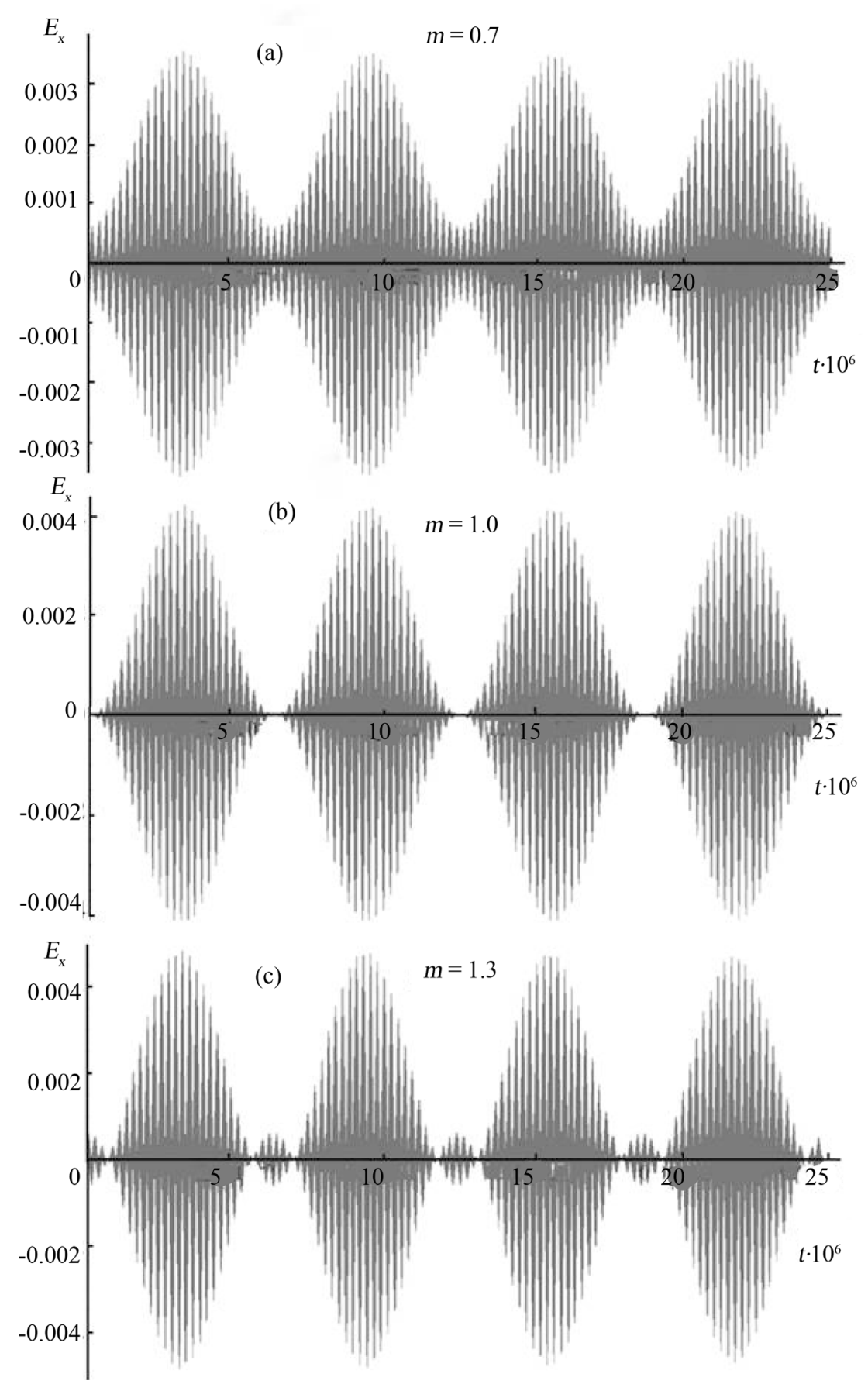

Figure 4. Graphs of integrated waves (components of the sine) for different values of the parameter $m$ : (a) $m=0.7$; (b) 1.0 ; and (c) 1.3 .

$$
\left.E\right|_{\Gamma}=\varphi(t) .
$$

Equations (43) and (44) are the initial conditions for the problem, (45) is the boundary condition $(39,40)$, and $\Gamma$ is the boundary of the domain. The function $E$ depends on three variables and is of the form $E(t, x, y)$. It is also a vector $\boldsymbol{E}=\left(E_{x}, E_{y}\right)$. The boundary $\Gamma$ of our domain is nothing but the boundary of a square. To solve this problem, we used the Wolfram Mathematics package. 


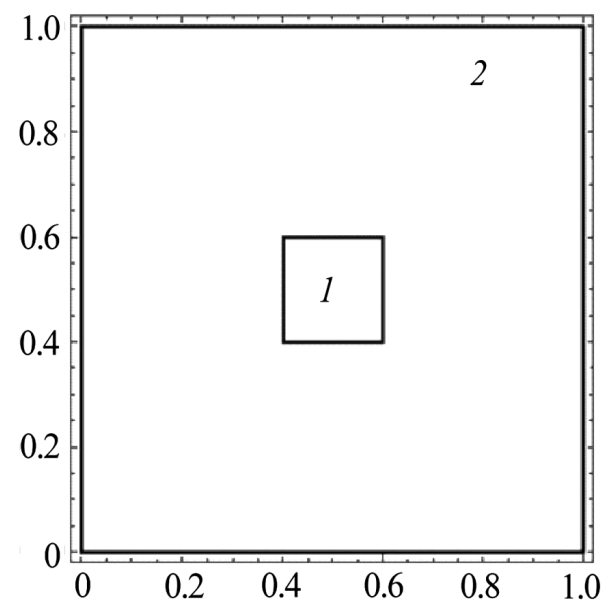

Figure 5. Problem solution domains: 1) copper bar; 2) domain with air.

The results obtained at $m=1$ for the multiplier $(1-\cos \theta t)$ and various instants of time are shown in Figure 6.

In conclusion, we note that the attempt to compare the results of numerical simulation of this problem in the Wolfram Mathematics and COMSOL Multiphysics environment was unsuccessful, because the task using the COMSOL Multiphysics package was not considered when specifying functions of the form (39), (40) on the boundaries. The calculation results are also difficult to compare, for example, with calculations based on difference schemes for the equations of oscillations of a string [25], since the problem is considered on a time interval $0<t \leq T$, i.e., the matching problem for $t=0$ is not considered. Attempts to calculate using the existing wave packets for a group of waves also failed, since the wave packet [26]

$$
\Psi(x, t)=C(x, t) \mathrm{e}^{i\left(\omega_{0} t-k_{0} x\right)}=2 C\left(k_{0}\right) \frac{\sin \left(\left[\left(\frac{\mathrm{d} \omega}{\mathrm{d} k}\right)_{0} t-x\right] \Delta k\right)}{\left(\frac{\mathrm{d} \omega}{\mathrm{d} k}\right)_{0} t-x} \mathrm{e}^{i\left(\omega_{0} t-k_{0} x\right)}
$$

does not satisfy the matching conditions at the initial time. Therefore, the Wolfram Mathematics package informs about the error in formulating the initial boundary-value problem. We note, however, that the numerical calculations in both packages qualitatively (increased concentration of the electromagnetic field on sharp edges) correspond to the experimental data provided that COMSOL Multiphysics uses harmonic functions at the boundary of the region without taking into account the time of signal establishment, but without transient processes.

\section{Conclusions and Suggestions}

A coordinated physicomathematical model for the propagation of a soliton-like electromagnetic pulse in a heterogeneous medium is developed in the presence 

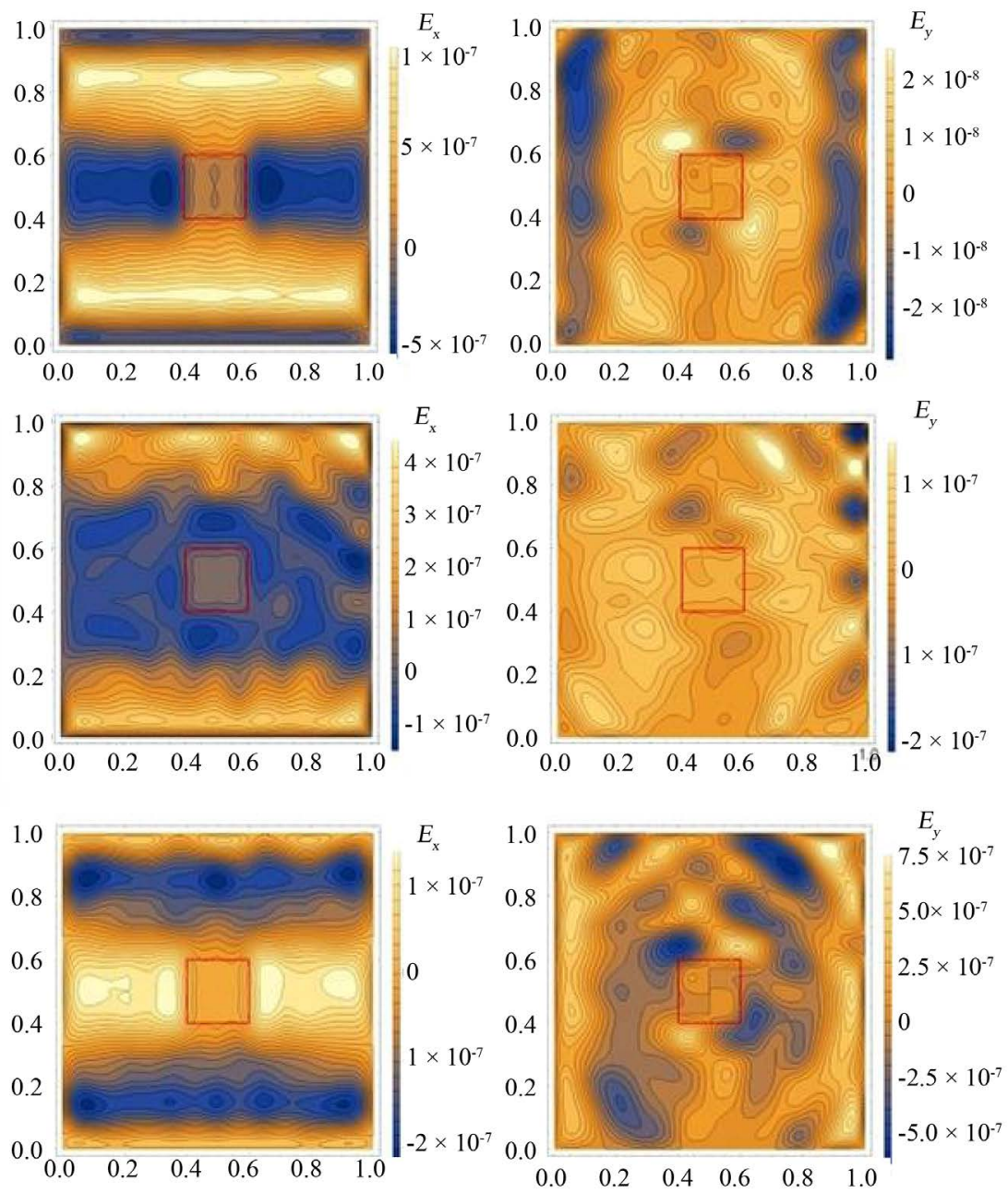

Figure 6. Distributions of the intensity in the region $\mathrm{m}=1$ for the multiplier $(1-\cos \theta t)$ and different instants of time: top, $t=4 \times 10^{-9}$; center, $t=6 \times 10^{-9}$; bottom, $t=8 \times 10^{-9}$.

of strong discontinuities in the electromagnetic field. The model is based on the reduction of Maxwell's equations to the well-studied wave equation. When the electromagnetic pulse was specified, its amplitude modulation was taken into account, as was the nonstationary broadening of the spectral line. The results obtained: the condition of continuity of the total current in a heterogeneous medium, a consistent soliton-like pulse of the electromagnetic field, allow us to justify the use of through-counting schemes for numerical analysis of the interaction of an electromagnetic field and a piecewise homogeneous body.

The method of the "non-sharp" boundary is substantiated using the Dirichlet theorem for piecewise smooth functions and the condition of continuity of the total current. Numerical simulation of the propagation of a soliton-like pulse signal in a medium with strong electromagnetic field discontinuities is performed with account taken of the broadening of the spectral line.

The work was supported by the Belarusian Republican Foundation for Basic Research, project No. F16K-075. 


\section{References}

[1] Grinchik, N.N. (2008) Simulation of Electrophysical and Thermophysical Processes in Porous Media. Nauka, Minsk, Belarus. (In Russian)

[2] Grinchik, N.N. (2000) Interaction of Thermal and Electric Phenomena in Polariged Media, Matemat. Modelirovanie, 12, 67-76.

[3] Grinchik, N.N. and Tsurko, V.A. (2002) Problem of Modeling of the Interaction of Nonstationary Electric, Thermal, and Diffusion Feilds in Layered Media. Journal of Engineering Physics and Thermophysics, 75, 693-699.

[4] Grinchik, N.N., et al. (2011) Fundament Problems of the Electrodynamics of Heterogeneous Media with Boundary Conditions Corresponding to the Total-current Continuity. Electromagnetic Wave Propagation in Complex Matter, INTECH, Rijeka.

[5] Grinchik, N.N. and Grinchik, Yu.N. (2012) Fundamenttal Problems of the Electrodynamics of Heterogeneous Media. Physics Research International, 2012, Article ID 185647.

[6] Grinchik, N.N. (2014) Electrodynamics of Inhomogeneous (Laminated, Angular) Structures. Journal of Electromagnetic Analysis and Applications, 6, 57-105. https://doi.org/10.4236/jemaa.2014.65009

[7] Stratton, J.A. (1948) The Theory of Electromagnetism. OGIZ, Moscow-Leningrad. (In Russian)

[8] Kalashnikov, S.G. (1985) Electricity. Nauka, Moscow. (In Russian)

[9] Tamm, I.E. (2003) Foundations of the Theory of Electricity. Fizmatgiz, Moscow. (In Russian)

[10] Purcell, E. (1971) Electricity and Magnetism. Nauka, Moscow. (In Russian)

[11] Irodov, I.E. (1983) Fundamental Lows of Electromagnetism. Vysshaya Shkola, Moscow. (In Russian)

[12] Zil’berman, G.E. (1970) Electricity and Magnetism. Nauka, Moscow. (In Russian)

[13] Telesnin, R.V. and Iakovlev V.F. (1969) A Course in Physics. Electricity. Prosveshchenie, Moscow. (In Russian)

[14] Sivukhin, D.V. (1996) Electricity, in Two Parts. Nauka, Moscow. (In Russian)

[15] Erofeenko, V.T. and Kozlovskaya, I.C. (2001) Partial Equations with Applications in Economics: A Course of Lectures. BGU, Minsk, Belarus. (In Russian)

[16] Sveshnikov, A.G., Bogolubov, A.N. and Kravtsov, V.V. (2004) Lectures on Mathematical Physics: A Textbook for University Students. Nauka, Moscow. (In Russian)

[17] Sobel'man, I.I. (1954) On the Theory of Width of Atomic Spectral Lines. Physics-Uspekhi, 54, 551-586. https://doi.org/10.3367/UFNr.0054.195412c.0551

[18] Vol'kenstein, M.V. (1951) Molecular Optics. Gostekhizdat, Moscow-Leningrad. (In Russian)

[19] DeWitt, C. and Blandin, A., Eds. (1966) Quantum Optics and Electronics: Lectures at Summer School of Theoretical Physics of the University of Grenoble, Les Houches, France. Mir, Moscow. (In Russian)

[20] Bronshtein, I.N. (2010) Reference Book of Mathematics for Engineers and University Students. Publishing House Lan, St. Petersburg. (In Russian)

[21] Silin, V.P. and Rukhadze A.A. (2013) Electromagnetic Properties of Plasma and Plasmalike Media. 3rd Edition, Publishing House Librokom, Moscow. (In Russian)

[22] Vakman, D.E. and Vainshtein L.A. (1977) Amplitude, Phase, Frequency-Fundamental 
Concepts of Oscillation Theory. Physics-Uspekhi, 20, 1002-1016. https://doi.org/10.3367/UFNr.0123.197712f.0657

[23] Evans, D.F. and Matesich, M.A. (1977) Measurement and Interpretation of Electrical Conductivity. In: Yeager, E. and Salkind, A.J., Eds., Measurement Methods in Electrochemistry, Vol. 2, Mir, Moscow. (In Russian)

[24] Antonov, V.F., Chernysh, A.M., Pasechnik, V.I., et al. (2000) Biophysics. Gumanitarnyi Izdatel'skii Tsentr "Vlados", Moscow. (In Russian)

[25] Samarskii, A.A. (1989) Theory of Difference Schemes. Nauka, Moscow. (In Russian)

[26] Blokhintsev, D.I. (2015) Quantum Mechanics: Lectures on Selected Issues. 2nd Edition, Lenand, Moscow. (In Russian) 